\title{
Screening And Brief Intervention For Diabetes
}

\author{
And Hypertension Among Hispanic Men \\ John E. Scarbrough, PhD, PT, RN, CNE; ; Joe Tomaka, PhD \\ aWestern New Mexico University, College Boulevard Silver City NM

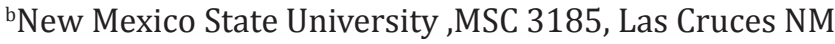 \\ john.scarbrough@wnmu.edu
}

\begin{abstract}
Along the U.S.-Mexico border, significant disparities exist in the incidence, prevalence, diagnosis, and treatment of diabetes mellitus and hypertension in the Hispanic population, especially males. The target population suffers from the sequelae of lack of long-term management to a greater degree than the general population. The first step in efforts to minimize the consequences of poor disease identification and management (cardiac disease, stroke, limb amputation, etc.) is to screen at-risk individuals and encourage their engagement with positive self-care behaviors. Early detection and effective interventions have the greatest ability to decrease existing morbidity and mortality disparities for Hispanic males in the U.S-Mexico border region.

The project utilized screening and brief intervention methodologies in locations convenient to the target population, seeking to increase both identification and follow-up care for Hispanic men with type 2 diabetes and hypertension. All participants were screened for type 2 diabetes, hypertension, and body mass. The experimental group received 1:1 motivational interviewing seeking to encourage follow-up and discussion of the results, and the control group received a standard, plain text printout of results with no motivational interview. Follow-up telephone calls and return of stamped, pre-addressed postcards were used to capture follow-up data.

The project identified significant incidence of type 2 diabetes and hypertension within the population and also validated correlation between the two conditions and obesity. Further research is indicated to enhance data capture upon conclusion the brief intervention, for which follow-up contact results were inconclusive due to limited follow-up participation.
\end{abstract}

Key words: Diabetes, Hypertension, Screening Brief Intervention, Hispanic Males

\section{INTRODUCTION}

Along the U.S.-Mexico border, significant disparities exist in the incidence, prevalence, diagnosis, and treatment of diabetes mellitus and hypertension in the Hispanic population, especially males. ${ }^{1}$ The target population of Hispanic males suffers from the sequelae of long- term lack of management to a greater degree than the general population. ${ }^{2}$ The first step in efforts to minimize the serious consequences of poor disease identification and management (cardiac disease, stroke, limb amputation, etc.) is to screen at-risk individuals and encourage their engagement with positive self-care behaviors.

The project applied innovative efforts to incorporate screening in convenient locations with the application of existing behavioral change promotion methodologies, together seeking to increase both identification and follow-up care for the identified conditions within the target population. ${ }^{3}$ The entire project was conducted in an attempt to challenge commonly-held beliefs of Hispanic men that "If I feel healthy, I am healthy." Use of Prochaska's Transtheoretical Change Model (TTM) ${ }^{4}$ provided the theoretical framework supporting the use of the structured intervention in seeking to modify the health behavior of participants. 


\section{Relevance}

Improvements in the screening methods used to identify the chronic diseases of diabetes mellitus and hypertension and the ability to intervene early in the disease processes in support of health care self-promotion offer tremendous opportunity to improve the health of the community. Early detection and effective interventions have the greatest ability to decrease existing morbidity and mortality disparities for Hispanic males in the U.S-Mexico border region.5

\section{METHODS}

\section{Overview}

The project sought to identify Hispanic men with newly discovered hypertension, prehypertension, diabetes or prediabetes and ask for their participation in a randomized, six- week study conducted at work-related sites in the local community. Half of the study participants received a novel intervention that paired personalized health feedback (PHF) with motivational interviewing; the other half received only simple feedback on their screening results. All participants were asked to

(a) speak with a close family member about their screening results and

(b) see a healthcare provider of their own choosing or one to which we could refer them.

Approximately six weeks later we contacted participants again to find out if they had completed these behaviors, and to assess changes in health beliefs and behavioral intentions from screening levels.

\section{Participants}

128 subjects comprised of men aged 18-82 years were recruited and screened, with a mean age of 43.5 years. All participants self-identified as Hispanic or Latino males. 111 of those screened met criteria for inclusion in the study; of those screened, only 17 were ineligible due to inability to meet inclusion criteria based on their assessment findings falling within normal ranges for all values. Entry criteria included a positive screening result for pre-diabetes or diabetes (i.e., HgbAlc of 5.7 or greater) or hypertension (SBP > 139 or DBP > 89). Anticipatory power analysis indicated the need to include a minimum of 88 participants in order to detect a statistically significant difference in means at post-test. Preparatory analyses suggested a minimum cell size of 37 per condition (control vs. experimental) to detect a medium effect size with a power of .80 and alpha set liberally at .10 . The final subject population of 111 participants met the required population sample size without difficulty. Of the 111 included in the study, 53 were randomized to the personalized feedback condition and 58 were randomized to the generic feedback condition.

\section{Setting}

The study took place at local establishments known for conducting business with working-class Hispanic men who were likely to be at risk for undiagnosed/untreated diabetes or hypertension. Such establishments included rock and gravel purveyors and a local flea market known to patronize a predominantly Hispanic population. The purposive component of the study therefore sought to bring screening and brief intervention to the targeted population that is underserved from a health-care perspective.

\section{Demographic}

Specific demographic data collected included: age, ethnicity, marital status, previous diagnosis of hypertension Y/N, previous diagnosis of diabetes mellitus $\mathrm{Y} / \mathrm{N}$, and a follow-up e-mail and telephone number information.

\section{Clinical Measurements}

Specific measures captured included, height, weight, blood pressure, pulse rate, and Hemoglobin A1C (glycosylated hemoglobin). Height and weight were obtained and recorded using a calibrated balance scale with stadiometer. Blood 
pressure measures were obtained through use of a Critikon Dinamap 8100 electronic blood pressure measuring device, and Hemoglobin A1C was measured using the Siemens DCA Vantage glucometer. All measures were obtained using standard medical procedures and sample collection techniques to ensure client safety.

\section{Health Beliefs}

The study assessed general beliefs about health at both the time of intervention and at the approximate six-week followup. Specific beliefs assessed included current health, health worry or concern, and attitudes toward going to the doctor based upon the Health Perceptions Questionnaire by Ware.6 We also employed a measure of behavioral intention to 1) see a healthcare professional, and 2) to speak with a spouse or other close relative about feedback received from participation in the study. Both intention measures were developed in line with recommendations by Ajzen.7

\section{Health Behaviors}

The study assessed two behavioral outcomes: whether the participant spoke with a (a) close relative and/or (b) healthcare professional. These behaviors were assessed 1) during a follow-up telephone interview conducted approximately six weeks after the intervention and 2) via receipt of self-addressed, stamped postcards, initialed by participants' healthcare professional and/or family member.

\section{Procedure}

As noted previously, the data-collection and intervention components of the project were conducted in community settings frequented by Hispanic men rather than in more traditional clinical agency screening locations established for the convenience of those collecting data. Data collection commenced only after receiving requisite Institutional Review Board (IRB) approval from the hosting university. Local businesses known for Hispanic male clientele were initially selected, specifically, landscaping materials. Participation at these sites, however, was marginal at best, with a preponderance of individuals reporting an inability to take time away from work to participate. As a result, the venue was relocated, keeping in mind the key purpose of making the project one easily accessible to the target population. A suitable site was identified at an indoor flea market that was heavily patronized by the local Hispanic population on weekends.

Screenings were conducted on Saturdays and Sundays with a high degree of success in recruiting applicants and yielding a rich pool of potential subjects. Participation in the project was encouraged through offering a potential total of \$25.00 in gift cards to participants meeting criteria and agreeing to participate. Data were collected in four-hour sessions scheduled on weekends, with each individual session requiring approximately 20 minutes for completion per subject.

The research team was comprised of community health workers (promotoras), some of whom were bilingual (English/ Spanish); bilingual staff were available on-site for all screenings. All of the workers were trained in the delivery of culturally competent health care and culturally competent conduct of research per Collaborative Institutional Training Institute (CITI) guidelines. The principal investigator (PI) was a licensed Registered Nurse who was responsible for supervising all screening and intervention activities. As described, the study was conducted at local places of business frequented by men who work in blue-collar, manual labor professions.

The research team worked together with hosting site managers and proprietors to secure areas near entrances where tables were set up to conduct the study. Study personnel recruited participants with the incentive of a $\$ 5$ gift card, a free record of their test results, and the possibility of an additional $\$ 20$ incentive (for a total of $\$ 25.00$ for full participation) if the participants qualified and agreed to be included in the larger study. Bilingual signs were set up explaining and recruiting participants to the study. Individuals who agreed to the screening phase completed an informed consent statement, a brief demographic and health history form, and a brief survey of health beliefs. Following completion of the forms, staff collected individual health data as previously outlined (blood pressure, pulse rate, HbA1C, weight, and height). 
Based on results, those not eligible for the study (i.e., no risk of diabetes or hypertension) received a simple printout of their test results and a $\$ 5.00$ gift card. Eligible participants next were randomized using a random numbers table to the Personalized Feedback with MI (PFMI) condition or the Simple Feedback (SF) condition.

Participants in the experimental (PFMI) condition received a personalized document outlining the findings of the various screening tests which was used as the forum to engage the individual in a brief motivational interview. The forms included information pertaining to the health conditions of diabetes, hypertension, and overweight/obesity. The forms also presented graphic displays of test results with clear demarcations of risk levels for each health parameter. The personalized feedback form included information on health risks associated with their condition and ways that participants could reduce their risks. In addition to receiving the detailed form itself, a trained promotora reviewed the form with the individual to (a) answer questions and ensure understanding, and (b) engage the individual in a brief motivational interview. The promotora asked open-ended questions, sought to enhance motivation for seeking health attention, and supported the participant's self-efficacy to seek medical treatment. The interactions were conducted embracing both cultural appropriateness and respect of individual autonomy, consistent with the philosophy of motivational interviewing.7 Staff training in MI was conducted by Tomaka (Co-I) who has received training in motivational interviewing and has extensive experience with brief motivational approaches.

Participants in the control (simple findings only) group received the screening results only along with information about where they could seek additional medical attention if desired. The form used to present feedback was similar to that used by medical laboratories i.e., observed values with normal ranges.

All participants in the study received two addressed, stamped postcards and were requested to return them after

(a) conversations about test results with a close relative or loved one and

(b) a visit to a health-care professional.

Finally, staff conducted follow-up interviews in approximately six weeks via telephone. Variables assessed at the sixweek post-screening follow-up included

(a) whether they discussed their health issues with a family member,

(b) whether they visited and/or contacted a health-care professional.

On-site procedures required a maximum of 20 minutes for each participant. Following participation in the screening, participants in both the experimental and control groups were thanked for their participation with an additional \$20.00 gift card incentive. All participants were provided contact information for appropriate health-care resources including facilities and health-care agencies who had agreed to function in this capacity at no initial cost to participants for any individuals who elected to pursue follow-up care based on assessment findings.

\section{RESULTS}

Data collection and results of analysis identified a high prevalence of hypertension and diabetes within the population, consistent with and/or exceeding anticipated findings as established in the literature. Further analysis of the aggregated data revealed that there were no statistically significant differences between the experimental and control groups concerning discussions with loved ones and/or scheduling of follow-up medical care. Correlation for experimental versus control groups compared with follow-up call resulted in Pearson's $r=0.035, p=0.713$. A total of 9 cards were returned, and all of the cards returned were from the control (non-intervention) condition, demonstrating Pearson's $r$ $=-0.025, p=-0.463$ for experimental versus control groups correlated with returned postcard. Due to the low rate of return of cards and/or response to follow-up telephone calls, meaningful analysis was rendered impossible. There was a statistically significant small correlation between BMI and HgbAlc (Pearson's $r=.213, p=.025$ ), as would be expected where predispositions towards glucose intolerance are exacerbated by increases in body weight and/or obesity. 


\section{Ancillary Findings}

For the entire sample of those surveyed (all participants), only 17 (13.28\%) were ineligible due to normal blood pressure and normal HgbA1c, demonstrating that $86.72 \%$ of those surveyed had abnormal BP or HgbA1c or both abnormal BP and HgbA1c concurrently. Mean results obtained are presented in Tables 1, 2, and 3.

Table1 Blood Glucose

\begin{tabular}{|l|l|l|l|l|l|}
\hline $\begin{array}{l}\text { \# of Subjects } \\
\text { (Total = 128) }\end{array}$ & $\begin{array}{l}\text { A1c }<\text { 5.7 } \\
\text { Normal }\end{array}$ & $\begin{array}{l}\text { A1c 5.7-6.4 } \\
\text { Impaired }\end{array}$ & $\begin{array}{l}\text { A1c >=6.5 } \\
\text { Diabetic }\end{array}$ & $\begin{array}{l}\text { A1c }>\mathbf{5 . 7} \\
\text { Impaired/ } \\
\text { Diabetic }\end{array}$ & A1c Range \\
\hline 92 & $71.88 \%$ & & & & $4.7-5.6$ \\
\hline 21 & & $16.41 \%$ & & & $5.7-6.49$ \\
\hline 15 & & & $11.71 \%$ & & $6.5-11.5$ \\
\hline $36(21+15)$ & & & & $28.12 \%$ & $5.7-11.5$ \\
\hline
\end{tabular}

Table2 Blood Pressure

Systolic Blood Pressure

\begin{tabular}{|l|l|l|l|l|l|}
\hline $\begin{array}{l}\text { \# of Subjects } \\
\text { (Total = 128) }\end{array}$ & $\begin{array}{l}\text { SBP }<\mathbf{1 2 0} \\
\text { Normal }\end{array}$ & $\begin{array}{l}\text { SBP 121-139 } \\
\text { Pre-Hypertension }\end{array}$ & $\begin{array}{l}\text { SBP >=140 } \\
\text { Hypertension }\end{array}$ & $\begin{array}{l}\text { SBP >120 } \\
\text { Pre/Hypertension }\end{array}$ & $\begin{array}{l}\text { SBP } \\
\text { Range }\end{array}$ \\
\hline 34 & $26.6 \%$ & & & & $87-120$ \\
\hline 48 & & $37.5 \%$ & & & $121-139$ \\
\hline 46 & & & $35.94 \%$ & & $142-216$ \\
\hline $94(48+46)$ & & & & $63.9 \%$ & $5.7-11.5$ \\
\hline
\end{tabular}

Diastolic Blood Pressure

\begin{tabular}{|l|l|l|l|l|l|}
\hline $\begin{array}{l}\text { \# of Subjects } \\
\text { (Total = 128) }\end{array}$ & $\begin{array}{l}\text { DBP<80 } \\
\text { Normal }\end{array}$ & $\begin{array}{l}\text { DBP 81-89 } \\
\text { Pre-Hypertension }\end{array}$ & $\begin{array}{l}\text { DBP >90 } \\
\text { Hypertension }\end{array}$ & $\begin{array}{l}\text { DBP >81 } \\
\text { Pre/Hypertension }\end{array}$ & $\begin{array}{l}\text { DBP } \\
\text { Range }\end{array}$ \\
\hline 75 & $58.59 \%$ & & & & $51-80$ \\
\hline 30 & & $23.43 \%$ & & & $81-89$ \\
\hline 23 & & & $17.96 \%$ & & $90-146$ \\
\hline $53(30+23)$ & & & & $41.41 \%$ & \\
\hline
\end{tabular}

Table3 BMI

\begin{tabular}{|l|l|l|l|l|l|c|}
\hline \# of Subjects & $\begin{array}{l}\text { BMI < 25 } \\
\text { Normal }\end{array}$ & $\begin{array}{l}\text { BMI 25-30 } \\
\text { Overweight }\end{array}$ & $\begin{array}{l}\text { BMI > 30 } \\
\text { Obese }\end{array}$ & $\begin{array}{l}\text { BMI > 25 } \\
\text { Overweight/ Obese }\end{array}$ & $\begin{array}{l}\text { Mean } \\
\text { BMI }\end{array}$ & BMI Range \\
\hline 8 & $6.25 \%$ & & & & 23.31 & $19.77-24.9$ \\
\hline 29 & & $22.65 \%$ & & & 27.88 & $25.06-29.77$ \\
\hline 75 & & & $58.59 \%$ & & 36.23 & $30.11-36.16$ \\
\hline $104(29+75)$ & & & & $81.24 \%$ & & \\
\hline
\end{tabular}

DISCUSSION

Closing data analysis was severely restricted due to the low return rate of cards and response to follow-up telephone surveys. This may be a problem with the study capture methodology more than the absolute lack of increased awareness 
and/or motivation to consider behavior modification on the part of subjects. In total, only 9 postcards were returned, all from the non- personalized feedback (control) group. Those who returned one card, returned both (Loved one and PCP). This is in contrast to a significantly larger number of participants who self-reported both conversation and/or PCP contact during the telephone follow up, yet failed to return either postcard provided.

Although BMI was not directly targeted by the study as a component of screening and participation or intervention, a preponderance of evidence relates overweight and/or obesity to both hypertension and impaired blood glucose metabolism. The data collected strongly supports the importance of addressing the targeted conditions of hypertension and impaired glucose metabolism, as it is clear that the majority those meeting screening criteria targeted by the study (HTN and HgbA1C) are either overweight or obese (70.7\%), thereby providing confirmation of correlation between abnormal glucose tolerance, hypertension, and BMI.

\section{Confounds}

The most surprising confound was one of difficulty in achieving follow-up within the sample population. Precise rationale for the low return rate remains difficult to ascertain. Modification of follow-up protocol of the study is recommended to promote capture of more accurate data. This belief is validated through a discrepancy between the low number of cards returned and higher number of verbal reports of behavior change upon telephone inquiry followup. The data showed an increased number of verbal reports of health care consultation or discussion with a loved one when contrasted with the lower return rate of stamped, pre-addressed cards. One possible modification to the protocol would be to encourage participation not only at the time of initial contact, but also upon receipt of follow-up materials.

\section{Implications for Future Study}

Future follow-up studies plan to modify the capture protocol portion of the study to provide pre- stamped, pre-addressed post cards which have an option for Yes or No, versus the implied Yes for receipt of card in order to promote clarity of response. As further incentive to improve the capture rates, a second, higher-value incentive (gift card) will be mailed to participants upon receipt of their completed card(s) and/or response to telephone inquiry.

\section{Summary}

Results of the study validated the current prevalence and incidence of hypertension, type 2 diabetes mellitus, and overweight/obesity in the target population. Results of the study were disappointing concerning an inability to "close the loop" with data capture and thereby determine any actual impact made by the motivational interviewing and alternative format presentation. It is possible that the study did achieve its goals of motivating Hispanic men to speak with family and/or loved ones concerning their health findings, but this was not demonstrated.

Planning for future deployments of the same protocol are currently underway addressing the same population, incorporating modifications as noted. The findings of this initial study support the need to continue efforts addressing the health needs of underserved Hispanic males in rural and border populations concerning the well-known consequences of uncontrolled hypertension, type 2 diabetes, and overweight/obesity.

\section{ACKNOWledgiments and Compliance With ETHical STANDARdS}

As the product of grant-funded research, the project presented met with no conflicts of interest across professional, academic, or other disciplines. Grant funding was received from the Mountain West CTR-IN as a pilot study (Prime award number 1U54GM104944-2, Sub-award number 15-746Q-NMSU-PG22-00.

All research was approved by the New Mexico State University institutional review board (IRB) and all procedures followed were in accordance with the ethical standards of the IRB and the Helsinki Declaration of 1975, as revised in 2000. Informed consent was obtained and documented in keeping with IRB requirements. NMSU IRB approval 11221-A, dated 7/2/2014.

The authors declare that there is no conflict of interest regarding the publication of this paper. 


\section{REFERENCES}

1. Shiller JS, Lucas JW, \& Peregoy JA. Summary health statistics for U.S. adults: National health interview survey, 2011. CDC Vital and Health Statistics. 2012;10(256).

2. Elder K, Meret-Hanke L, Dean C, et al. Men's health: Disparities in confidence to manage health. Int J Mens Health. 2013;12(3):260-275.

3. Clark M, Hampson SE, Avery L, \& Simpson R. Effects of a brief tailored intervention on the process and predictors of lifestyle behavior changes in patients with type 2 diabetes. Psychol Health Med. 2004;9(4):440-449. doi: 10.2105/AJPH.84.5.783

4. Prochaska JO \& DiClemente CC. The transtheoretical approach: Crossing traditional boundaries of change. 1984; Homewood IL: J. Irwin.

5. Fryar CD, Hirsch R, Eberhardt MS, Yoon SS, \& Wright JD. Hypertension, high serum total cholesterol, and diabetes: Racial and ethnic prevalence differences in U.S. adults, 1999-2006. NCHS Data Brief. 2010; 36. Hyattsville, MD: National Center for Health Statistics.

6. Ware JE. Scales for measuring general health perceptions. Health Serv Res. 1976:396-415. Available from: http://www.ncbi.nlm.nih.gov/pmc/articles/PMC1071941/pdf/hsresearch00553-0068.pdf

7. Ajzen I. Constructing a theory of planned behavior questionnaire. Available from http://people. umass.edu/ aizen/pdf/tpb.measurement.pdf

8. Miller W, \& Rollnick S. Motivational interviewing: Preparing people for change. New York, NY: The Guilford Press; 2002

Citation: John E. Scarbrough, Screening And Brief Intervention For Diabetes And Hypertension Among Hispanic Men American Research Journal of Nursing Volume 2016; pp:1-7

Copyright (c) 2016 John E. Scarbrough, This is an open access article distributed under the Creative Commons Attribution License, which permits unrestricted use, distribution, and reproduction in any medium, provided the original work is properly cited. 